\title{
Treatment of esophageal achalasia in children: Today and tomorrow
}

\author{
Tamara Caldaro ${ }^{\mathrm{a}, *}$, Pietro Familiari ${ }^{\mathrm{b}}$, Erminia Francesca Romeo ${ }^{\mathrm{a}}$, Giovanni Gigante ${ }^{\mathrm{b}}$, Michele Marchese ${ }^{\mathrm{b}}$, \\ Anna Chiara Iolanda Contini a , Giovanni Federici di Abriola ${ }^{a}$, Salvatore Cucchiara ${ }^{c}$, Paola De Angelis ${ }^{\text {a }}$, \\ Filippo Torroni a , Luigi Dall'Oglio a , Guido Costamagna ${ }^{\mathrm{b}}$ \\ a Digestive Surgery and Endoscopy Unit, Bambino Gesù Children's Hospital, Rome, Italy \\ ${ }^{\mathrm{b}}$ Digestive Surgery and Endoscopy Unit, Catholic University, Rome, Italy \\ c Pediatric Gastroenterology and Liver Unit, Sapienza University, Rome, Italy
}

\section{A R T I C L E I N F O}

\section{Article history:}

Received 19 January 2015

Accepted 13 February 2015

Available online $\mathrm{xxxx}$

Key words:

esophageal achalasia

esophageal manometry

Heller myotomy

POEM

\begin{abstract}
A B S T R A C T
Background: Esophageal achalasia (EA) is a rare esophageal motility disorder in children. Laparoscopic Heller myotomy (LHM) represents the treatment of choice in young patients. Peroral endoscopic myotomy (POEM) is becoming an alternative to LHM.

The aim of this study is to evaluate the effectiveness, safety, and outcomes of POEM vs LHM in treatment of children with EA.

Methods: Data of pediatric patients with EA, who underwent LHM and POEM from February 2009 to December 2013 in two centers, were collected.

Results: Eighteen patients ( 9 male, mean age: 11.6 years; range: $2-17$ years) were included. Nine patients (6 male, mean age: 10.7 years; range: $2-16$ years) underwent LHM, and the other 9 ( 3 males, mean age: 12.2 years; range: $6-17$ years) underwent POEM procedure.

Mean operation time was shorter in POEM group compared with LHM group (62/149 minutes). Myotomy was longer in POEM group than in LHM group $(11 / 7 \mathrm{~cm})$. One major complication occurred after LHM (esophageal perforation). No clinical and manometric differences were observed between LHM and POEM in follow-up. The incidence of iatrogenic gastroesophageal reflux disease was low (1 patient in both groups).

Conclusions: Results of a midterm follow-up show that LHM and POEM are safe and effective treatments also in children. Besides, POEM is a mini-invasive technique with an inferior execution timing compared to LHM. A skilled endoscopic team is mandatory to perform this procedure.
\end{abstract}

(c) 2015 Elsevier Inc. All rights reserved.
Esophageal achalasia (EA) is a rare, chronic and progressive motility disorder, manometrically characterized by absence of esophageal peristalsis and incomplete or absent relaxation of the lower esophageal sphincter (LES) [1].

Pediatric achalasia is often misdiagnosed and may result in debilitating symptoms and failure to thrive.

The estimated incidence of EA in children is 0.11 per 100,000 , without racial or gender predilection [2]. Although clinical, radiological and endoscopic results may raise the suspicion of EA, the definitive diagnosis depends on esophageal manometry.

Laparoscopic Heller myotomy (LHM) is the recommended procedure for adults with EA, while medical treatments and/or endoscopic esophageal pneumatic dilations are usually reserved for those older than 45 years and with high surgical risk [1].

\footnotetext{
* Corresponding author at: Digestive Surgery and Endoscopy Unit, Bambino Gesù Children's Hospital, Piazza S. Onofrio 4, 00165, Rome, Italy. Tel.: + 39 0668592841; fax: + 390668592949

E-mail address: tamara.caldaro@opbg.net (T. Caldaro).
}

Peroral endoscopic myotomy (POEM) is a minimally invasive procedure, that has been recently developed and introduced in clinical practice for the treatment of EA in adults [3-5].

POEM combines the benefits of an endoscopic procedure with the long-term efficacy of a surgical myotomy. Preliminary studies demonstrated that POEM is associated with a low rate of complications and a good effectiveness at midterm follow-up [6].

Up to now, isolated cases of children with EA have been treated with POEM, with excellent results at short-term follow-up [7,8].

The aim of this multicenter study is to compare the perioperative outcomes and clinical efficacy of POEM and LHM for the treatment of EA in children.

\section{Patients and methods}

Eighteen pediatric patients with EA, who had undergone LHM or POEM between 2009 and 2014, were retrospectively identified from the prospectively collected databases of two tertiary referral centers: Bambino Gesù Children's Hospital (Digestive Surgery and Endoscopy Unit) and Gemelli University Hospital (Digestive Surgery and Endoscopy Unit). 
Preoperatively, barium X-ray series, esophageal manometry and upper endoscopy were performed.

The Eckardt score system was used for the clinical evaluation before and after both procedures [9].

Since the introduction of POEM at Gemelli University Hospital in 2012, patients with EA and their parents were informed regarding the two available options (LHM and POEM) to freely decide which procedure to choose.

The surgical treatment (LHM) was performed in 9 patients (mean age at surgery: 10.8 years; range: $2-17$ years) by the same team of three surgeons at Bambino Gesù Children's Hospital; while a single endoscopist performed POEM at Gemelli University Hospital in the other 9 children (mean age at POEM: 12.2 years; range: $6-17$ years).

\subsection{Laparoscopic Heller myotomy (LHM)}

The patient is placed in the supine position and 4 trocars (one trocar: $10 \mathrm{~mm}$; 3 trocars: $5 \mathrm{~mm}$ ) are inserted in to the abdomen under direct visualization.

The esophagogastric junction is exposed for $6-8 \mathrm{~cm}$ proximally and the myotomy is performed longitudinally for a minimum of $6 \mathrm{~cm}$, using a blunt dissector and scissors.

To prevent burning or perforation of the esophageal mucosa, the use of hook cautery or cautery scissors was avoided.

To exclude small esophageal perforations, air is inflated into the stomach using a nasogastric tube while a sodium chloride solution is introduced into the abdomen. An esophageal perforation is detected when bubbles can be seen into the abdomen.

The procedure is completed by performing a Dor fundoplication.

\subsection{Peroral endoscopic myotomy (POEM)}

POEM procedure is performed according to the technique described by Inoue et al [10] in 2010. Broad spectrum antibiotics (usually a cephalosporin or amoxicillin) are administered preoperatively An upper endoscopy is performed using a high-definition endoscope (GIF-H180J; Olympus Tokyo, Japan) with carbon dioxide $\left(\mathrm{CO}_{2}\right)$ insufflation. The esophageal mucosa is cut on the anterior wall using an endoscopic cautery knife (Triangle-tip knife, Olympus), approximately 10-12 cm above the esophageal gastric junction (Fig. 1A). After the mucosal incision is completed, the esophageal submucosa is dissected using a spray coagulation current (VIO300D, ERBE Elektromedizin $\mathrm{GmbH}$, Tubingen, Germany) (Fig. 1B). A long tunnel is created in the esophageal submucosa extending from the mucosal incision to $3 \mathrm{~cm}$ along the anterior gastric wall (Fig. 1C). The esophageal muscular layer is exposed and cut through the submucosal tunnel. The myotomy includes the circular bundles of the muscular layer and extends for $2-3 \mathrm{~cm}$ up the gastric wall (Fig. 1D). At the end of the procedure, the mucosal incision is closed using endoscopic clips (EZ Clips, Olympus). Twenty-four or 48 hours after myotomy, an upper endoscopy under general anesthesia and a gastrografin esophageal study are performed to exclude mucosal tears or leaks; then patients are fed a soft diet.

\subsection{Follow-up, data collection and analysis}

Demographics, data on the clinical history and the results of preoperative studies (upper endoscopy, barium swallow and esophageal manometry) were collected from the medical charts and the prospective databases, recorded and compared.

Information on the perioperative outcomes, including procedure time, length of myotomy, perioperative complications and length of hospitalization, was analyzed.

After the procedures, patients underwent a rigorous follow-up.

Esophageal manometry was repeated after 6 months.

The onset of gastroesophageal reflux disease (GERD) was detected by $\mathrm{pH}$-monitoring and upper endoscopy, 6 months postoperatively.

The Eckardt score was used to evaluate the clinical efficacy of the procedures during follow-up. a

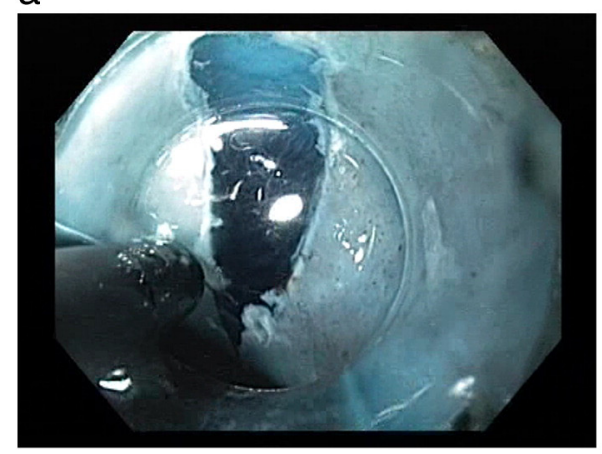

C

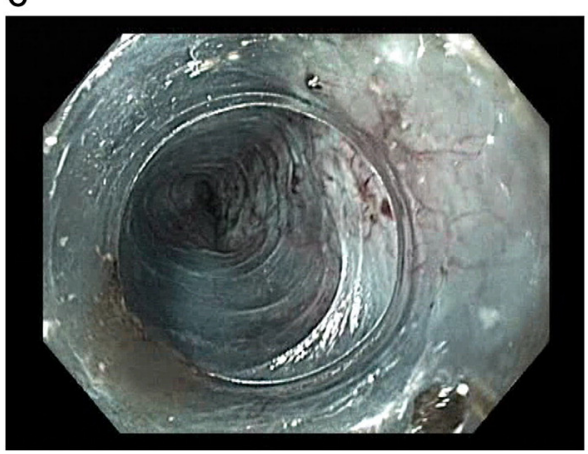

b

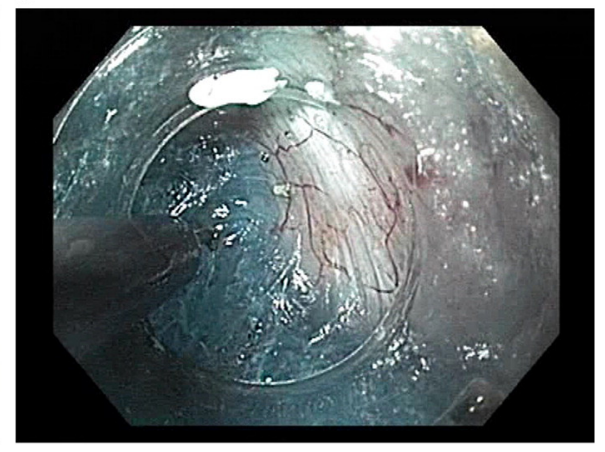

d

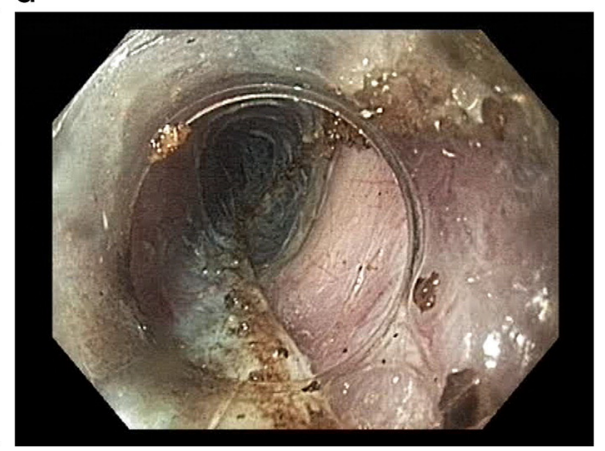

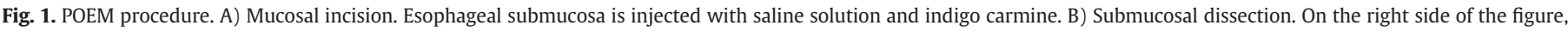

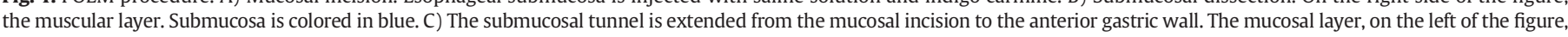
is intact. D) Myotomy begins about $3 \mathrm{~cm}$ distal to the mucosal incision and it is extended until the anterior gastric wall. 
Therapeutic failure was defined by a value major than 3 .

Ethical approval for this study was obtained from our ethics board (protocol number: $255 \mathrm{LB}$ ).

Informed written parental and patient consents were acquired before performing upper endoscopy, esophageal manometry, $\mathrm{pH}$-monitoring and surgical or endoscopic procedures.

\subsection{Statistical analysis}

Student's t-test for unpaired sample was used to compare mean procedure time, length of myotomy, length of hospitalization, preoperative and postoperative lower esophageal sphincter (LES) pressure between the two groups of patients.

Student's t-test for paired sample was used to evaluate preoperative and postoperative LES pressure of patients in each group.

Wilcoxon rank sum test and Wilcoxon signed rank test were used to compare preoperative and postoperative Eckardt score between the groups and in each group, respectively.

A p value $<.05$ was considered statistically significant.

\section{Results}

In the last 4 years, a total of 18 children ( $M: F=9: 9$; mean age at EA treatment:11.6 years; range: $2-17$ years) with a diagnosis of EA were treated.

Since 2009, 9 patients (M:F $=6: 3$; mean age at surgery: 10.8 years; range: 2-17 years) have undergone LHM and Dor fundoplication in Bambino Gesù Children's Hospital.

Since 2012, 9 children (M:F = 3:6; mean age at POEM: 12.2 years; range: $6-17$ years) have been treated with POEM procedure in Gemelli University Hospital.

Baseline characteristics of our population are reported in Table 1.

Preoperatively, upper endoscopy excluded other pediatric congenital or acquired diseases that could have lead to dysphagia (congenital esophageal stenosis and peptic strictures); whereas barium swallow study showed a typical "bird's beak" narrowing in all children of both groups, but no presence of megaesophagus.

At baseline, the Eckardt score was 7.8 (range: 6-12) in the LHM group and 7 (range: $4-10)$ in the POEM group $(\mathrm{p}=0.512)$.

The preoperative mean LES resting pressure was $38.48 \pm 9.13 \mathrm{mmHg}$ and $45.32 \pm 24.06 \mathrm{mmHg}$ in the LHM and in the POEM cohort, respectively $(\mathrm{p}=0.006)$.

Before LHM and POEM, 3/9 (33.3\%) patients in the LHM group (early onset of EA in 2 patients; severe malnutrition and weight loss in 1 patient) and $1 / 9(11.1 \%)$ patient in the POEM group had received pneumatic dilations; in the LHM group, one child had taken calcium channel blockers (nifedipine) until surgery.

Mean operating times were significantly shorter in the POEM group compared with the LHM group ( $62 \pm 12.7$ minutes vs $149 \pm$ 33.8 minutes, $\mathrm{p}<0.01$ ).

Myotomy was longer in the POEM group than in the LHM group $(11 \pm 2 \mathrm{~cm}$ vs $7 \mathrm{~cm} \pm 1.5 \mathrm{~cm}$ ), but the difference was not statistically significant $(\mathrm{p}=0.26)$.

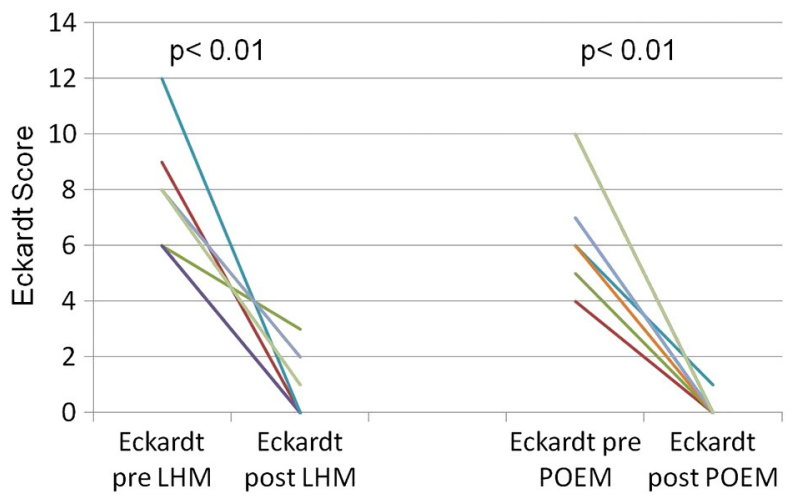

Fig. 2. Preoperative and postoperative Eckardt score in LHM group and in POEM group.

One major complication occurred in the LHM group which was perforation of the esophageal mucosal layer. This was suspected by the onset of fever and abdominal pain on the first postoperative day, and confirmed by a gastrografin esophageal study.

Reoperation was necessary to repair the esophageal perforation.

In the POEM cohort, 1 patient required Veress needle placement to decompress pneumoperitoneum; another patient had a small esophageal mucosal tear that was clipped. No postoperative complications occurred.

Oral intake was started on the third (range: 1-12) postoperative day in the LHM group and after 2.3 (range: $1-3$ ) postoperative days in the POEM group ( $\mathrm{p}<0.01)$.

The average postoperative hospital stay was $6 \pm 4.9$ days (range: $3-18$ ) in the LHM group and $4.1 \pm 1.4$ days (range: $2-7$ ) in the POEM group $(\mathrm{p}<0.01)$.

\subsection{Postoperative outcomes}

The mean follow-up was 31 months (range: 13-63) in the LHM group and 12.7 months (range: 5-28) in the POEM group.

In terms of symptomatic outcomes, follow-up was not significantly different in the 2 groups and all patients experienced a marked improvement in symptom scoring.

Eckardt score changes between preoperative and postoperative data were statistically significant both in the LHM group (pre-LHM Eckardt score $=7.8$, post-LHM Eckardt score $=1, \mathrm{p}<0.01$ ) and the POEM group (pre-POEM Eckardt score $=7$, post-POEM Eckardt score $=0$, $\mathrm{p}<0.01$ ) (Fig. 2).

On the contrary, the differences in the postoperative Eckardt score between LHM group and POEM group were not statistically significant (LHM group vs. POEM group: $\mathrm{p}=0.07$ ).

The comparison between treatments is summarized in Table 2.

During follow-up, esophageal manometry was repeated in 6/9 (66.6\%) patients in the LHM group and in $7 / 9$ (77.7\%) patients in the POEM group.

When preoperative and postoperative manometric data were compared, both LHM and POEM resulted in statistically significant decreases

Table 1

Baseline characteristics of study population.

\begin{tabular}{|c|c|c|}
\hline & LHM group & POEM group \\
\hline Patients & 9 & 9 \\
\hline Gender (male:female ratio) & $6: 3$ & $3: 6$ \\
\hline Age at treatment (mean $\pm S D$, range) & $10.8 \pm 5.4$ years (range: $2-17$ ) & $12.2 \pm 3.8$ years (range: $6-17$ ) \\
\hline Associated disease & Allgrove syndrome (1 patient) & Pilocytic astrocytoma (1 patient) \\
\hline \multirow[t]{2}{*}{ Previous treatments } & 3/9 (33.3\%) pts: PD & 1/9 (11.1\%) pt: PD \\
\hline & 1/9 (11.1\%) pt: Calcium blockers & \\
\hline
\end{tabular}

LHM: laparoscopic Heller myotomy; POEM: peroral endoscopic myotomy

PD: pneumatic dilations

Pts: patients 
Table 2

Comparison of procedure-related parameters and adverse events between LHM and POEM group.

\begin{tabular}{|c|c|c|c|}
\hline & LHM & POEM & P value \\
\hline Operative time (mean $\pm \mathrm{SD}$, minutes) & $149 \pm 33.8$ & $62 \pm 12.7$ & $<0.01$ \\
\hline Myotomy length $($ mean $\pm \mathrm{SD}, \mathrm{cm})$ & $7 \pm 1.5$ & $11 \pm 2$ & $=0.26$ \\
\hline Postoperative oral intake (mean $\pm \mathrm{SD}$, days, range) & $3 \mathrm{rd} \pm 3.7$ (range: $1-12$ ) & 2 nd \pm 0.8 (range: $1-3$ ) & $<0.01$ \\
\hline Postoperative hospital stay (mean $\pm \mathrm{SD}$, days, range) & $6 \pm 4.9$ (range: $3-18)$ & $4.1 \pm 1.4$ (range: $2-7)$ & $<0.01$ \\
\hline Early complications and treatments & -Esophageal perforation ( $1 \mathrm{pt})$ : reoperation. & $\begin{array}{l}\text {-Pneumoperitoneum (1pt): Veress needle placement. } \\
\text {-Mucosal perforation ( } 1 \mathrm{pt}) \text { : clips. }\end{array}$ & \\
\hline Long-term complications and treatments & $\begin{array}{l}\text { - Dysphagia ( } 2 \text { pts): Pneumatic dilations } \\
\text { GERD (1 patient): PPI }\end{array}$ & GERD (1 patient): PPI & \\
\hline
\end{tabular}

GERD: gastroesophageal reflux disease

PPI: proton pump inhibitors

Pt: patient

of LES resting pressure (pre-LHM mean LES resting pressure $=38.48 \pm$ $9.13 \mathrm{mmHg}$, post-LHM mean LES resting pressure $=14 \pm 10.7 \mathrm{mmHg}$, $\mathrm{p}<0.01$; pre-POEM mean LES resting pressure $=45.32 \pm 24.06 \mathrm{mmHg}$, post-POEM mean LES resting pressure $=19.27 \pm 6.57 \mathrm{mmHg}, \mathrm{p}=$ 0.03) (Fig. 3).

In the LHM group, 2 children needed esophageal pneumatic dilations, one after three months and the other two years after surgery.

\subsection{LHM, POEM and GERD}

To diagnose GERD, upper endoscopy and $\mathrm{pH}$-monitoring were performed in 6/9 (66.6\%) patients both in the LHM and in the POEM group.

$\mathrm{pH}$-monitoring was positive in one patient in each cohort. Upper endoscopy documented macroscopic esophagitis in one patient in the POEM group. Proton pump inhibitors were administered in the two cases.

\section{Discussion}

In the pediatric population there are no defined guidelines for the treatment of EA.

The available therapeutic options are palliative managements, all directed at reducing patients' symptoms, improving esophageal emptying and preventing the formation of a megaesophagus.

Endoscopic pneumatic dilations are recommended in adulthood because they are effective, easy to perform, repeatable and low cost, but there's no shared consensus about their employment in children.

Di Nardo et al. [11] reported a good success rate after the first pneumatic balloon dilation in 24 children with EA. However in other pediatric studies a high rate of symptom recurrence, requiring further intervention, was described. [12-14].

In our study, pneumatic dilations were performed preoperatively only on selected patients with severe malnutrition and/or early

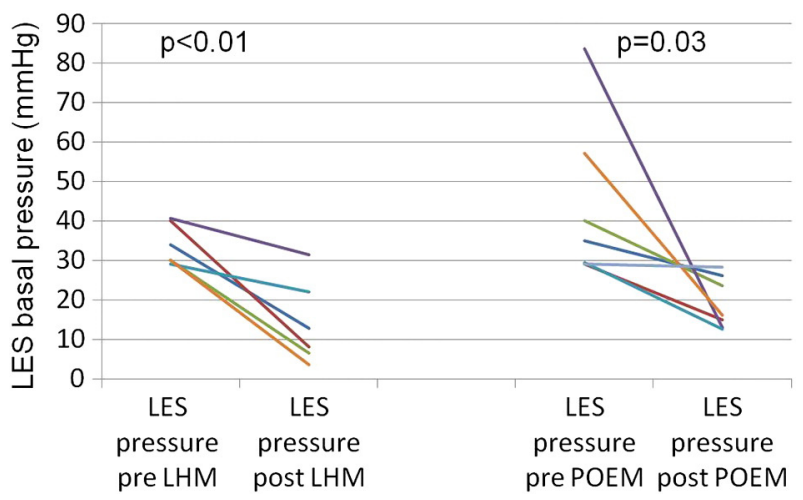

Fig. 3. Lower esophageal sphincter (LES) basal pressure before and after LHM and POEM. (before 2 years of age) onset of EA, in order to complete the diagnostic assessment.

According to the literature, Heller myotomy is considered the treatment of choice for children, as it provides more durable outcomes in comparison to pneumatic dilations in long-term follow-up $[15,16]$. The increasing use of laparoscopy, with the well-known advantages of shorter hospital stay, minor use of analgesics, faster return to daily activities and better cosmetic result, has further encouraged the application of the Heller myotomy [17-19].

Recently, the development of new minimally invasive techniques has revived the debate on the treatment of EA. The introduction of POEM, that incorporates the endoscopic approach with the principles of the natural orifice transluminal endoscopic surgery (NOTES) to perform a myotomy in the adult population, has found broad consensus among skilled endoscopists.

In the last few years, more and more endoscopy centers have begun to adopt POEM to treat EA in adulthood, with good short-term outcomes $[4,20,21]$.

The procedure has only recently been tested in pediatric age group: likely owing to the rarity of the disease in children, the need to first validate the technique in adults and the required learning curve of the endoscopist. In fact, if a learning curve of 20 has been proposed for adults, a longer experience could be mandatory to treat children [22].

A few pediatric cases have been described to date. Among the related studies, Familiari et al. ( 3 children) and Chen et al. ( 21 children) are the most reliable $[8,23]$.

Ours, is the first pediatric series that compares the conventional laparoscopic technique and the POEM procedure.

Our data demonstrated that POEM was characterized by shorter operative times, a longer myotomy, a lower rate of complications, faster time to feeding and more rapid discharge.

One of the main advantages of this technique is the ability to modifying the length of the myotomy, according to the preoperative manometric and endoscopic data.

As a matter of fact, in the POEM group the length of myotomy was almost doubled with respect to LHM group, but this difference did not affect the clinical outcomes, that were similar in the two cohorts, probably because surgical myotomies had a minimum total length of $6-7 \mathrm{~cm}$, as literature recommends, and this extension is enough to make them all successful [24].

POEM is considered a safe technique with minimal adverse events [25]. Subcutaneous and/or mediastinal emphysema, pneumoperitoneum and pneumothorax are common conditions related to air or $\mathrm{CO}_{2}$ insufflation during the procedure, which are normally selflimited or easily resolved [10]. $\mathrm{CO}_{2}$ is preferably employed instead of room air because it has the advantage of faster diffusion and quicker absorption [25].

Even in all our cases, POEM was completed with a low rate of minor complications, such as pneumoperitoneum or small perforations of the mucosal esophageal flap, without conversion to laparoscopy or open surgery. 
In the LHM cohort, on the contrary, one serious complication (perforation of the esophageal mucosa by burning) occurred, owing to a malfunction of the electro cautery device. So, since then on, our surgical approach was modified by eliminating the hook cautery.

This severe adverse event, however, influenced the postoperative outcomes of our study. In fact, if data of the patient with the mucosal perforation are excluded, times of refeeding and discharge of POEM group are very similar to the ones of LHM population.

In midterm follow-up, both procedures seem efficacious in ensuring an excellent clinical and functional outcome and the effect of myotomy on LES resting pressure is quite stable over time, regardless of the technique used.

To guarantee a more appropriate comparison between the two procedures, a wider clinical and manometric follow-up considering the possibility of clinical changes over time, would be necessary.

Currently, the major issues in debate are the utilization of the POEM technique in low-weight subjects and the risk of iatrogenic GERD after the procedure.

As regards the first topic, age and weight should not be considered as a limitation to the usage of POEM, as Maselli et al. demonstrated, treating a three-year patient affected by EA, Downs syndrome and severe malnutrition [7]. Consequently, the endoscopic technique seems to be applicable with the same equipment even in very young patients, but at the moment the available data on the matter are not sufficient to establish specific weight and age limits.

Referring to GERD, its possible onset after POEM, due to the impracticality of performing a fundoplication, prompts some criticism. Some authors suggest that the advantage of POEM in this procedure is the selective myotomy of the internal circular muscular fibers without dissection of the diaphragmatic hiatus and division of the crura, typically required to perform an adequate surgical myotomy [26].

Swanstrom et al. reported a GERD rate of $46 \% 6$ months after POEM [27], while in the series described by Teitelbaum et al., symptomatic GERD was present in only $17 \%$ of patients [28].

In our population, the rate of iatrogenic GERD was very low and similar both in POEM and LHM, but two important biases could limit this comparison between the two procedures.

The first is the small series of patients enrolled in each group, which could reduce the reliability of the study on this specific question.

The second is the difference in follow-up length between the two groups, which could underestimate the onset of GERD in POEM cohort.

Therefore, multicenter prospective randomized trials, assessing long-term outcomes, would be extremely useful.

In conclusion, clinical and functional outcomes of pediatric patients treated by LHM and POEM are excellent and are similar at midterm follow-up.

The age of patients is not a contraindication to POEM technique, though a skilled endoscopic team is mandatory to safely perform the procedure.

If this POEM is not available LHM remains the treatment of choice for EA in children.

However, both POEM and LHM should be performed in high-volume endoscopic and pediatric surgical centers of excellence, to delimit intraoperative and postoperative complications and to ensure the best longterm results.

\section{Conflict of interest}

None.

\section{Acknowledgements}

The authors thank:

- Prof. Geoffrey Blair, for the scientific support

- Dr. Maria Felicia Villani, for the statistical analysis

- Prof. Paolo Costantini, for the linguistic review

\section{References}

[1] Vaezi MF, Pandolfino JE, Vela MF. ACG clinical guideline: diagnosis and management of achalasia. Am J Gastroenterol 2013 Aug;108:1238-49.

[2] Mayberry JF, Mayell MJ. Epidemiological study of achalasia in children. Gut 1988;29: 90-3.

[3] Pasricha PJ, Hawari R, Ahmed I, et al. Submucosal endoscopic esophageal myotomy: a novel experimental approach for the treatment of achalasia. Endoscopy 2007;39:761-4.

[4] von Renteln D, Inoue H, Minami H, et al. Peroral endoscopic myotomy for the treatment of achalasia: a prospective single center study. Am J Gastroenterol 2012;107:411-7.

[5] Costamagna G, Marchese M, Familiari P, et al. Peroral endoscopic myotomy (POEM) for oesophageal achalasia: preliminary results in humans. Dig Liver Dis 2012;44:827-32.

[6] Stavropoulos SN, Desilets DJ, Fuchs KH, et al. Per-oral endoscopic myotomy white paper summary. Gastrointest Endosc 2014;80:1-15.

[7] Maselli R, Inoue H, Misawa M, et al. Peroral endoscopic myotomy (POEM) in a 3 years old girl with severe growth retardation, achalasia, and Down asyndrome. Endoscopy 2012;44:E285-7.

[8] Familiari P, Marchese M, Gigante G, et al. Peroral endoscopic myotomy for the treatment of achalasia in children. J Pediatr Gastroenterol Nutr 2013;57:794-7.

[9] Eckardt VF, Aignherr C, Bernhard G. Predictors of outcome in patients with achalasia treated by pneumatic dilation. Gastroenterology 1992;103:1732-8.

[10] Inoue H, Minami H, Kobayashi Y, et al. Peroral endoscopic myotomy (POEM) for esophageal achalasia. Endoscopy 2010;42:265-71.

[11] Di Nardo G, Rossi P, Oliva S, et al. Pneumatic balloon dilation in pediatric achalasia: efficacy and factors predicting outcome at a single tertiary pediatric gastroenterology center. Gastrointest Endosc 2012;76:927-32.

[12] Walton JM, Tougas G. Botulinum toxin use in pediatric esophageal achalasia: a case report. J Pediatr Surg 1997;32:916-7.

[13] Ip KS, Cameron DJ, Catto-Smith AG, et al. Botulinum toxin for achalasia in children. J Gastroenterol Hepatol 2000;15:1100-4.

[14] Pastor A, Mills J, Marcon MA, et al. A single center 26-year experience with treatment of esophageal achalasia: is there an optimal method? J Pediatr Surg 2009; 44:1349-54.

[15] Lee CW, Kays DW, Chen MK, et al. Outcomes of treatment of childhood achalasia. J Pediatr Surg 2010;45:1173-7.

[16] Franklin Ashanti L, Petrosyan Mikael, Kane Timothy D. Childhood achalasia: a comprehensive review of disease, diagnosis and therapeutic management. World J Gastrointest Endosc 2014;6:105-11.

[17] Pachl MJ, Rex D, De Coppi P, et al. Paediatric laparoscopic Heller's cardiomyotomy: a single centre series. J Pediatr Surg 2014:49:289-92.

[18] Douard R, Gaudric M, Chaussade S, et al. Functional results after laparoscopic Heller myotomy for achalasia: a comparative study to open surgery. Surgery 2004;136: $16-24$.

[19] Dempsey DT, Kalan MM, Gerson RS, et al. Comparison of outcomes following open and laparoscopic esophagomyotomy for achalasia. Surg Endosc 1999;13:747-50.

[20] Ujiki MB, Yetasook AK, Zapf M, et al. Peroral endoscopic myotomy: a short term comparison with standard laparoscopic approach. Surgery 2013;154:893-900.

[21] Bhayani NH, Kurian AA, Dunst CM, et al. A comparative study on comprehensive, objective outcomes of laparoscopic Heller myotomy with per-oral endoscopic myotomy (POEM) for achalasia. Ann Surg 2014 Jun;259:1098-103.

[22] Kurian AA, Dunst CM, Sharata A, et al. Peroral endoscopic esophageal myotomy: defining the learning curve. Gastrointest Endosc 2013;77:719-25.

[23] Chen WF, Zhou PH, Li QL, et al. Clinical impact of peroral endoscopic myotomy for achalasia of pediatric patients: a prospective single center study. Endoscopy 2012; 44(Suppl. 1):A11.

[24] Stefanidis D, Richardson W, Farrell TM, et al. SAGES guidelines for the surgical treatment of esophageal achalasia. Surg Endosc 2012;26:296-311.

[25] Ren Z, Zhong Y, Zhou P, et al. Perioperative management and treatment for complications during and after peroral endoscopic myotomy (POEM) for esophageal achalasia (EA) (data from 119 cases). Surg Endosc 2012;26:3267-72.

[26] Mittal RK, Balaban DH. The esophagogastric junction. N Engl J Med 1997;336: 924-32.

[27] Swanstrom LL, Kurian A, Dunst CM, et al. Long-term outcomes of an endoscopic myotomy for achalasia: the POEM procedure. Ann Surg 2012;256:659-67.

[28] Teitelbaum EN, Rajeswaran S, Zhang R, et al. Peroral esophageal myotomy (POEM) and laparoscopic Heller myotomy produce a similar short-term anatomic and functional effect. Surgery 2013;154:885-92. 